\title{
Movie Success Prediction ANd PERFORMANCE COMPARISON USING VARIOUS STATISTICAL APPROACHES
}

\author{
Manav Agarwal, Shreya Venugopal, Rishab Kashyap and R Bharathi \\ Department of CSE, PES University, Bangalore, India
}

\begin{abstract}
Movies are among the most prominent contributors to the global entertainment industry today, and they are among the biggest revenue-generating industries from a commercial standpoint. It's vital to divide films into two categories: successful and unsuccessful. To categorize the movies in this research, a variety of models were utilized, including regression models such as Simple Linear, Multiple Linear, and Logistic Regression, clustering techniques such as SVM and K-Means, Time Series Analysis, and an Artificial Neural Network. The models stated above were compared on a variety of factors, including their accuracy on the training and validation datasets as well as the testing dataset, the availability of new movie characteristics, and a variety of other statistical metrics. During the course of this study, it was discovered that certain characteristics have a greater impact on the likelihood of a film's success than others. For example, the existence of the genre action may have a significant impact on the forecasts, although another genre, such as sport, may not. The testing dataset for the models and classifiers has been taken from the IMDb website for the year 2020. The Artificial Neural Network, with an accuracy of 86 percent, is the best performing model of all the models discussed.
\end{abstract}

\section{KEYWORDS}

Regression Models, Clustering Techniques, Time Series Model, Artificial Neural Network, Movie Success, Statistical Significance.

\section{INTRODUCTION}

Movies are one of the most important contributing factors to the entertainment industry in the world today, and from a commercial perspective are among the highest revenue-generating businesses [1]. Hence from a movie industry point of view, it is important to know whether a movie that they are thinking of making will be successful. There are plenty of factors such as the genre of a movie, the popularity of a movie based on its votes, critical judgement, and so on which influence the choices made by people to watch certain movies of their liking. Thus, to identify a movie that is worth watching, people look into various websites and articles such as Metacritic, Rotten Tomatoes, IMDb and many more sites that give us the rating of the movie. Thus, the analysis involves the study of these user ratings and the other factors that affect the movie and this helps us identify whether a movie is truly successful or not. This would help those who are creating movies make better movies and thus get more revenue. It will also make sure that the audience gets to watch movies that they enjoy. We wish to exploit the various techniques and tools used to uncover useful information from a variety of data that provides information about movies to infer various useful traits in it using which we would like to build a movie success predictor. The data is used in predictive models which include regressors, classifiers and time series models. Each of these models uses the attributes associated with the movies as independent values used to predict the outcome rating of the movie. Crossing a certain pre-set 
threshold classifies it as a success, else we deem the movie unsuccessful. The models that we use consist of regression models such as Linear Regression, Logistic Regression, Ridge and LASSO Regression. Classifiers include machine learning models such as K-Means and Support Vector Machine. An Artificial Neural Network is also used. The final model that we use is Time Series analysis which allows us to observe the variations that take place over the years on the movie ratings and to identify trends and seasonality in the data if it exists. Hence the main contributions of this work are:

1. Find the kind of model that predicts the success of a movie the best.

2. Perform a comparative study between various models and also look into the statistical significance of each model.

3. Understand what features influence the success or failure of a movie.

4. Test it against the data of 2020 , the data available for movies that are being released recently.

5. Determine if the attributes for the model are easily available.

\section{LiteratURE SURVEY}

Before the literature survey, we had to analyze and select the right dataset to be used for this purpose through which we concluded on using the IMDb Extensive dataset [2]. This is accompanied by movies and their attributes scraped from the IMDb website for those released in the year 2020 through data mining techniques [3-4]. As shown by Abidi et. al [1] the next set of steps would be to identify the list of attributes that assist in predicting how popular a movie is through a thorough investigation into all the properties of a given movie. . They mention that movie genres contribute a vital role in the popularity of the movie because the movie industry firmly makes decisions on what type of movie customers of different ethnic groups liked, rate, and favoured the movie. Thus, to study such attributes and use them for prediction, we utilize a wide range of regression models [5] and Machine Learning techniques [6-7] to carry out our predictions, including the use of Neural Networks [8] for more efficient classification. To add value to our experiments, we perform a comparative study similar to that done by Dhir and Raj [9] who have compared specifically for machine learning models. Before that, we check the credibility of a model by performing various statistical tests [12-13] whose comprehensive explanation is the highlight of our paper. The best performing model in this paper gives an accuracy of $86 \%$ which holds good in comparison to the works done by Sharma et al [14] and the models used by Dhir and Raj [9].

\section{Proposed Methodology}

From the literature survey, we have gathered information about the various technologies and methods used to predict how successful a movie is. With reference to them, we build on some of the important models used and prove how significant they are by applying various statistical tests on them. Figure 1 shows the phases of our experiment along with the models we are testing. Data is first collected from various sources that can be either from pre-existing datasets that are curated by someone else or by scraping it directly from the internet. Through studying the statistical significance of the models we aim to prove the validity and merit of each type of model. In doing so we deduce the best model that can be used to predict the success rate of a given movie with the best performance. In the entire process, we use python programming language for our analysis as the libraries provided for statistical analysis allows for a much smoother and efficient process. 


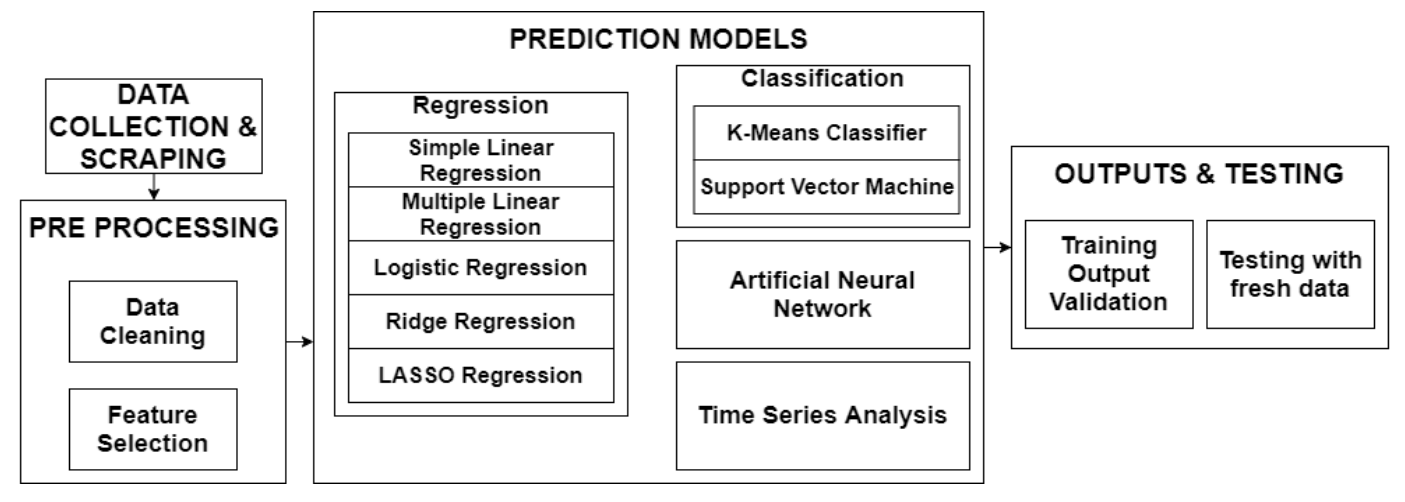

Figure 1. Proposed Methodology

As can be seen in Figure 1 the entire project is divided into 4 phases namely Data Collection, PreProcessing, Building the prediction models and the testing phase. In the Data collection phase we collected data from two sources, a pre-existing Extensive IMDb dataset from Kaggle which was used as the training and the validation dataset for the models and the other part of the dataset was obtained by scraping data off of the IMDb website which formed the testing dataset for these models. This is to ensure that whatever models that were made work well in a realistic scenario with the attributes that are available in the present day and will continue to work properly in the future. In the next phase, which is the Pre-Processing phase, we clean the data from both the sources and convert them to a form that can be used to develop and train the models. The next phase involves building various models using the dataset that has been created and testing them on the scraped dataset. In the next phase, we put the models that have been built through the testing dataset and perform statistical tests on them to check their validity.

\section{Dataset Description}

As mentioned above, this project uses the IMDb dataset [2]. This dataset consisted of 4 files movie related data such as budget, duration and so on, the data that give the number of votes or ratings for each movie for multiple demographics, data that involves biographical details of those that are involved in the movie-making industry like their place of birth, spouse etc and finally another file to act as a mapping between these files. Not all of the attributes present in the dataset were taken into consideration for the analysis. Some of them were filtered out based on the relevance of the problem at hand. For example, attributes related to the personal lives of the people involved in the film industry were not required for predicting whether a movie would be successful. Attributes such as the personalities in the movie were not used since they are categorical and we wanted to restrict this analysis to remain as numeric as possible. Some of the major attributes that were a part of the data include the movie rating itself, followed by the genre, top voter ratings, total votes, duration of the movie and the release date. Here, the genre is the only categorical variable that was used in the analysis since the number types of the genre is 21 which is less compared to the number of actors or directors or other personalities. A total of 81274 movies have been obtained from the dataset, and the values are first cleaned in the preprocessing stage before it is split into training and testing datasets. The split is done with respect to the year of release of a given movie. Web scraping has also been used to extract data from the IMDb official website, from which we obtain all the movies released in the year 2020, which is combined with the previous testing dataset to create the final validation dataset. 


\section{Pre-Processing}

The first step of pre processing is to remove all null values and outliers through the process of elimination, or replacement by mean or median values if necessary. The attribute "budget" had values in different kinds of currencies, which would have caused problems if they were used asis. Hence, for this analysis, the movies that are considered are only in US Dollars. One of the most important pre processing techniques used is the Multi Label Binarizer [15] which allows us to encode attributes having more than one feature such as the genre. To do this, we list out all the sub-features of the genre attribute as columns and for each movie, we put 1 if the movie belongs to that genre, else we leave it as 0 by default. This process is repeated for all movies until we have a list of numeric values depicting the genres that belong to the movie and those that do not. This way the genre which previously represented a one to many relationship mapping with the help of one attribute gets converted into a better representation with discrete form, making it easy for classification. Movies that range from the years 1990 to 2015 are grouped together to create the training dataset, and movies from 2015 to 2020 are considered as our testing dataset. Movies older than 1990, were too old to use in a model for predicting movies that are made today because there was a lot of difference in the social environment. Besides, the data for older movies was very scarce, incomplete compared to the data entries for newer movies. To implement our classification, we have chosen the "metascore" value from the dataset to act as our dependent variable for our research. This attribute represents the overall numeric rating of any given movie between 0 to 100, 0 being the worst possible score and 100 being the best possible score that a movie can receive. To make the classification easier, we group the metascore values into three types of clusters known as "bins" [16], where each bin represents a flop, a mediocre and a successful movie. Movies that have a metascore from 0 to 33 are considered to be a flop, those that have a metascore from 33 to 66 mediocre and those that have a metascore that is between 66 and 100 is considered as a hit. All the models used are tuned to output the predicted metascore values based on the categories created here.

\section{Feature Selection}

Once the data is pre-processed, feature selection is performed to determine which attributes from our pre-processed dataset can go into the respective model. This process to a great extent depends on trial-and-error, that is, taking a random subset of features and seeing how the model performs and if that performance is better or worse than another group of attributes. However, there are some formal ways of tackling this too, some of which have been employed in this analysis. The first is using the values that were obtained by performing a correlation analysis for the numeric attributes in the dataset to make a decision on which set of attributes best work together. For each pair of numerical attributes, Pearson correlation is used. When selecting features for a model it is important that those features must have a good correlation with the dependent variable which is the "metascore" and also, those features that are being used as independent variables for a model should not be correlated with one another. This situation leads to multi-collinearity. Next comes the use of the Variance Inflation Factor (VIF) which for a given set of attributes shows if they can be used together in a model. The subset of attributes that must go into the VIF analysis is determined by trial and error. All the models in this work except for those that use regularization techniques such as Ridge and Lasso regression used the correlation analysis and VIF for its feature selection process. 


\section{REGRESSION METHODS}

Regression is a technique that uses the Ordinary Least Squares method to predict certain values given certain inputs. The outputs of regression models with the exception of Logistic Regression are usually continuous estimates of the dependent variable. They are not used for classification. But since the problem that we are looking into involves the classification of a movie into Successful, Unsuccessful and Mediocre, the values predicted from the regression models need to be binned into the 3 categories before testing for accuracy.

\subsection{Simple Linear Regression}

The first type of model we look into would be the Linear Regression model using a single variable. Simple Linear Regression (SLR) is a statistical model in which there is only one independent variable and the functional relationship between the dependent variable and the regression coefficient is linear[12]. The regression line is of the form:

$$
Y_{i}=\beta_{0}+\beta_{1} X_{i}+\varepsilon_{i}
$$

Here, the dependent variable $Y_{i}$ is known as the outcome or response variable, and $X_{i}$ Is known as the independent variable for the $\mathrm{i}^{\text {th }}$ term, and the beta coefficients are known as the regression parameters. The final value-added is known as the random error value or the residual that is obtained on performing the regression. In our case, we use the metascore as our dependent variable. We make use of the Variance Inflation Factor or VIF to calculate the multicollinearity among all the attributes present. Only one attribute has to be selected since SLR involves only a single independent variable, and hence we choose the attribute having the highest VIF value. From the list of features, we choose the top1000_voters_rating having the highest VIF value as our independent variable. The SLR model is trained using this attribute for all movies. As seen from Figure 2, budget has the lowest VIF value of 0, while top1000_voters_ratings is the highest with a value of 0.55 .

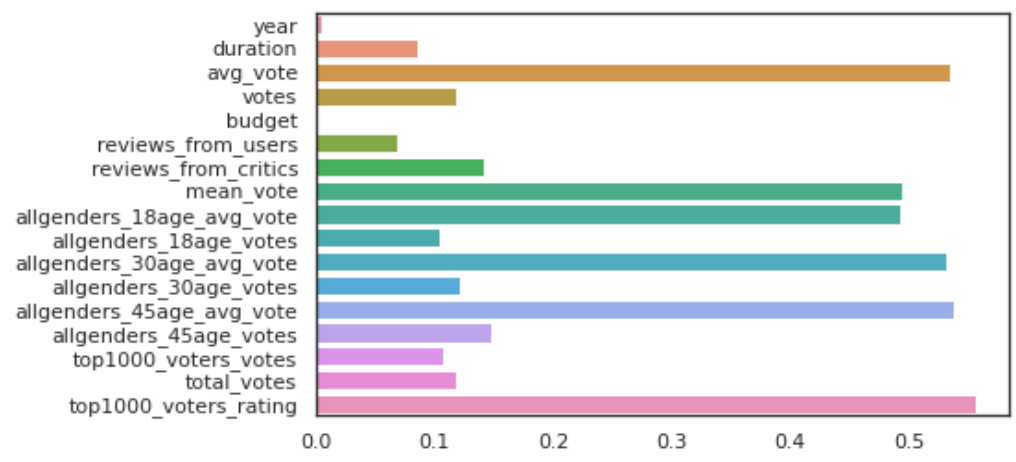

Figure 2. VIF distribution for the list of features

Once we have our trained model, we use the testing dataset to predict the future values, and then make a comparison between the true and predicted values. The accuracy of the model is calculated using a confusion matrix, which checks whether the values have truly been classified based on their values or falsely classified. Tests for statistical significance are performed on the model on completion of training. . On completing the proof of statistical significance, we move on to testing the model with the 2020 dataset, which yields an accuracy of 0.5833 . The only difference in this is the fact that we take the avg_vote value in-place of the top1000_voters_rating. 


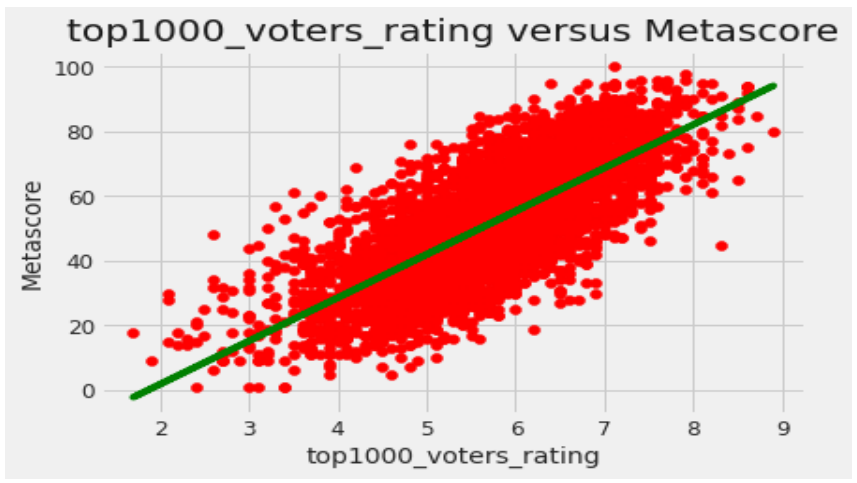

Figure 3. SLR model

\subsection{Multiple Linear Regression}

In a Multiple Linear Regression (MLR) model, there are many independent variables as opposed to the single independent variable in the SLR to estimate the target variable which is the metascore in this case. For this model, there are a total of sixteen attributes including the "top1000_voters_rating" along with twelve attributes that are the dummy variables generated by the Multi Label Binarizer for the genre. The VIF test again determines which features to select, and OLS tests, as well as the hypothesis tests like the Jarque Bera test and Lagrange's Multiplier test, are performed to check for statistical significance. This model has an accuracy of 0.7116 . Figure 4 shows a three-dimensional view of the MLR model using a sample of two of the independent variables along with the metascore. For the 2020 dataset, the same model is used giving an accuracy of 0.608 .
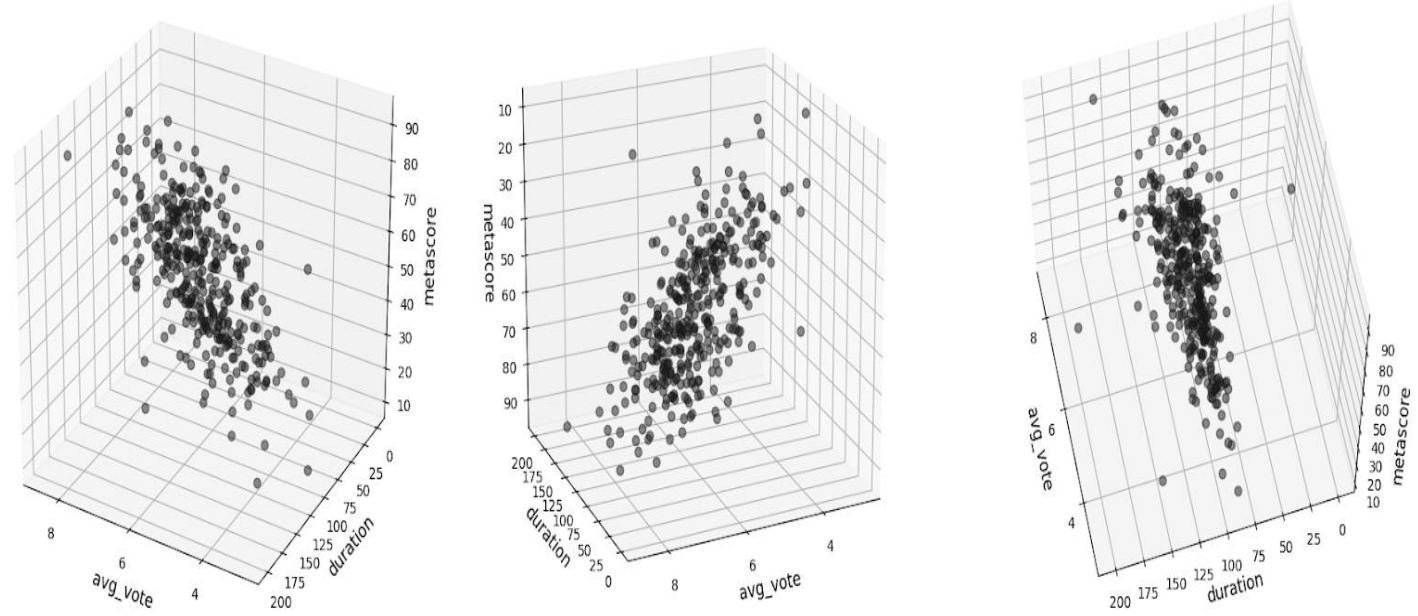

Figure 4. MLR model

\subsection{Logistic Regression}

Logistic Regression is a non-linear regression technique that is used when a set of independent variables are used to make a binary classification[13]. The main aim is to find the conditional probability of the occurrence of the event which facilitates the classification. Here, there is a slight change from the original steps that were performed for the target variables where now the metascore will be binned into two categories which are successful and unsuccessful instead of 
three. The model gives an accuracy of 0.76 . The ROC-AUC curve plotted from the confusion matrix of the results is shown in Figure 5. A Wald's test is performed to check the statistical significance of each of the independent variables used. This is similar to performing a t-test in the MLR model [12]. Pseudo $\mathrm{R}^{2}$ values are used to compare the intercept-only model to the model with the independent variables as it is not possible to calculate $\mathrm{R}^{2}$ directly. The analysis of the 2020 dataset for Logistic Regression gives an accuracy of 0.6833 .

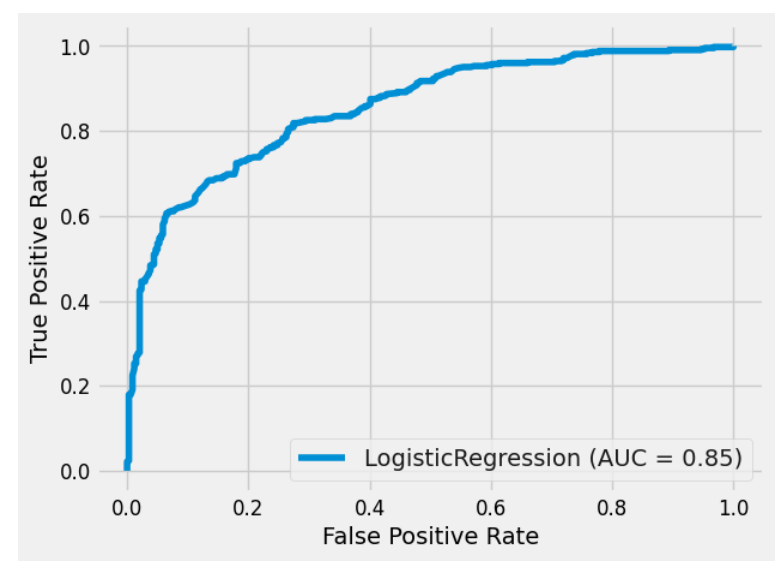

Figure 5. Plot to show the ROC-AUC curve

\subsection{Regularization Techniques}

Regularization in a regression model is a technique that prevents overfitting in the model by penalizing the coefficients involved in the regression equation. Ridge regression is a model that falls under the category of regularization techniques. It follows the L2 regularization method that reduces the summation of squared values of coefficients to the regression equation as penalties, which helps to penalize the multi collinearity [12]. The regularization parameter is set at 1150 for Ridge Regression, and the model gave us an accuracy of 0.74 analysing. LASSO regression on the other hand is a regularization technique that follows the L1 norm. This means that it adds the absolute value of coefficients as penalties to the cost function. We perform the same procedure as we did in the case of Ridge regression, where the regularization parameter is set to 0.145 , and we obtain an accuracy of 0.72 for this model. To test both the models, we once again use the 2020 testing dataset to confirm our results, obtaining an accuracy of 0.6, and we further perform statistical tests to prove their significance as displayed in the Results section.

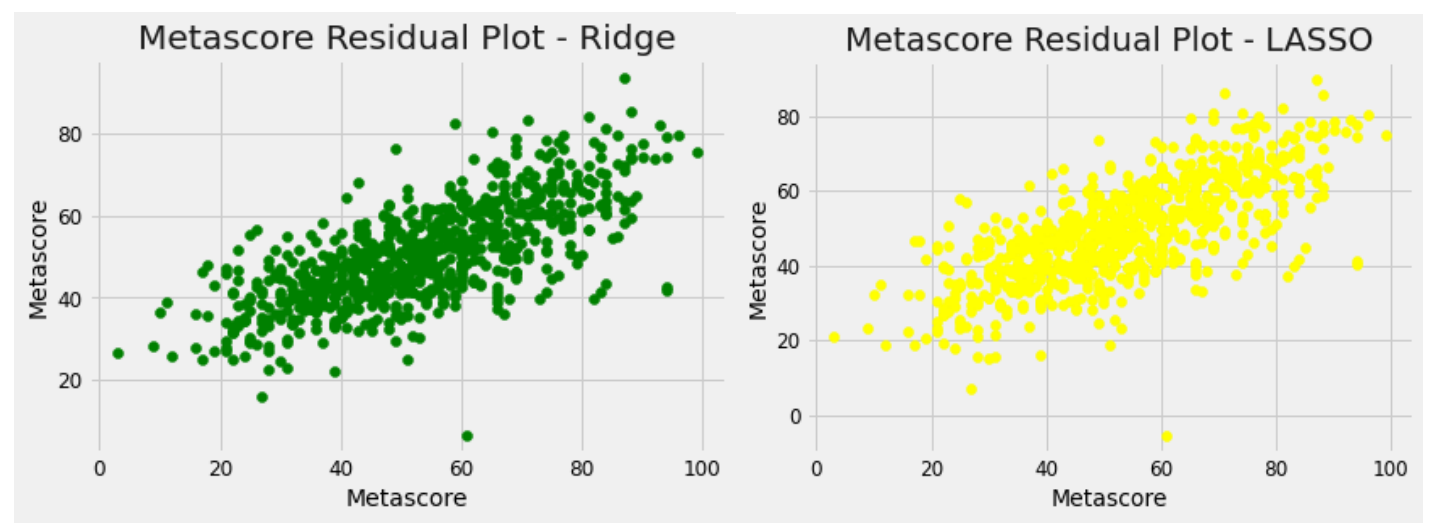

Figure 6. Residual plots for Ridge regression (left) and LASSO regression (right) 


\section{Classification Methods}

Unlike the regression models, the classification model outputs don't require binning before analysis for accuracy. Here a supervised classifier (SVM) and an unsupervised clustering technique (K-Means) is used for solving the movie success prediction problem.

\subsection{K-Means Classifier}

K-Means clustering is a non-hierarchical clustering method in which the number of clusters $(\mathrm{K})$ is decided a priori, in other words, decided prior to the execution of the model [12]. In our study for this paper, it has been used as a classifier where each cluster is considered as a separate class. In this case, we change the value of $\mathrm{K}$ to 3 to represent each of the metascore bins mentioned earlier [12]. On plotting the model, we obtain a final accuracy of 0.5. To prove the statistical significance, we perform the silhouette test, which is a technique used to evaluate the quality of the clusters created by the K-Means method, which calculates how similar each cluster is by size by checking the intra-cluster point distance and inter-cluster distances. The plot shown in Figure 7 shows the clusters created on testing the model using the 2020 dataset, from which we obtain an accuracy of 0.42 .

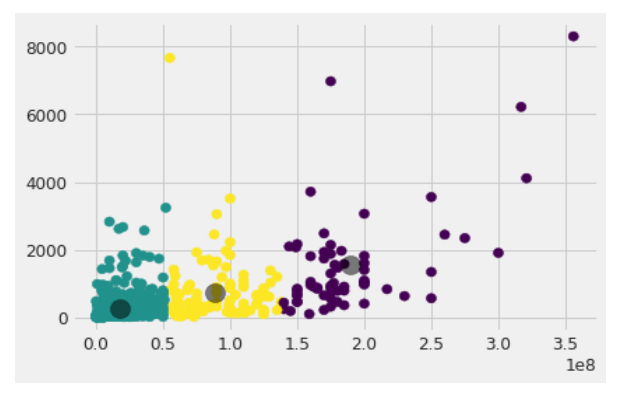

Figure 7. Centroid positions for KMeans Clustering

\subsection{Support Vector Machine}

The Support Vector Machine or SVM is a very powerful clustering technique that uses hyperplanes to distinguish between clusters. Traditionally, we use a single hyperplane to do this, but in our case we have made use of two hyperplanes to divide the outcome into three clusters, each corresponding to the three categories made to the metascore values. This model results in an accuracy of 0.71 using the training dataset, which is then used against the 2020 testing dataset to obtain an accuracy of 0.62 . As a proof of statistical significance, a silhouette test is performed to check whether the model's clusters are of good quality as we had done earlier for the K-Means classifier.

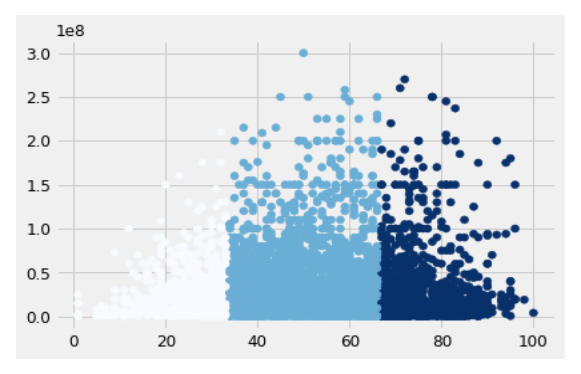

Figure 8. Plot to depict the cluster formation in SVM 


\section{Time SERIES ANALYSIS}

Forecasting is a process that uses past and present values to predict the values for the future. This is done by analysing and obtaining patterns from the data such as trend, seasonality and so on. These serve as the parameters of the model and help in defining what kind of model needs to be used for the time series analysis. For this problem statement, the date on which the film is released is taken as the primary variable that is being under analysis. On plotting the metascore value against the date_published attribute to get the time-series graph for the entire dataset and conducting some statistical tests we obtain Figure 9 and Figure 10.

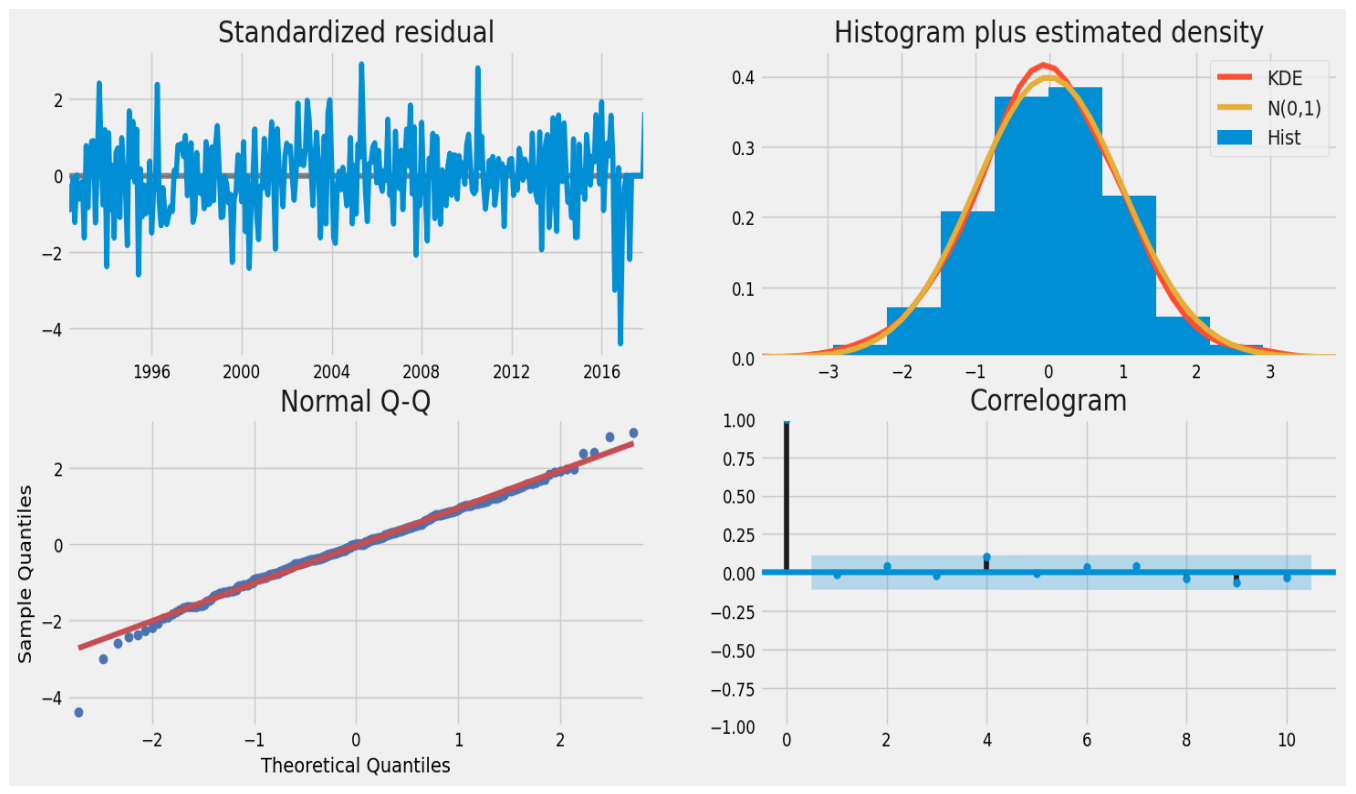

Figure 9. Various plots for statistical significance of time series model

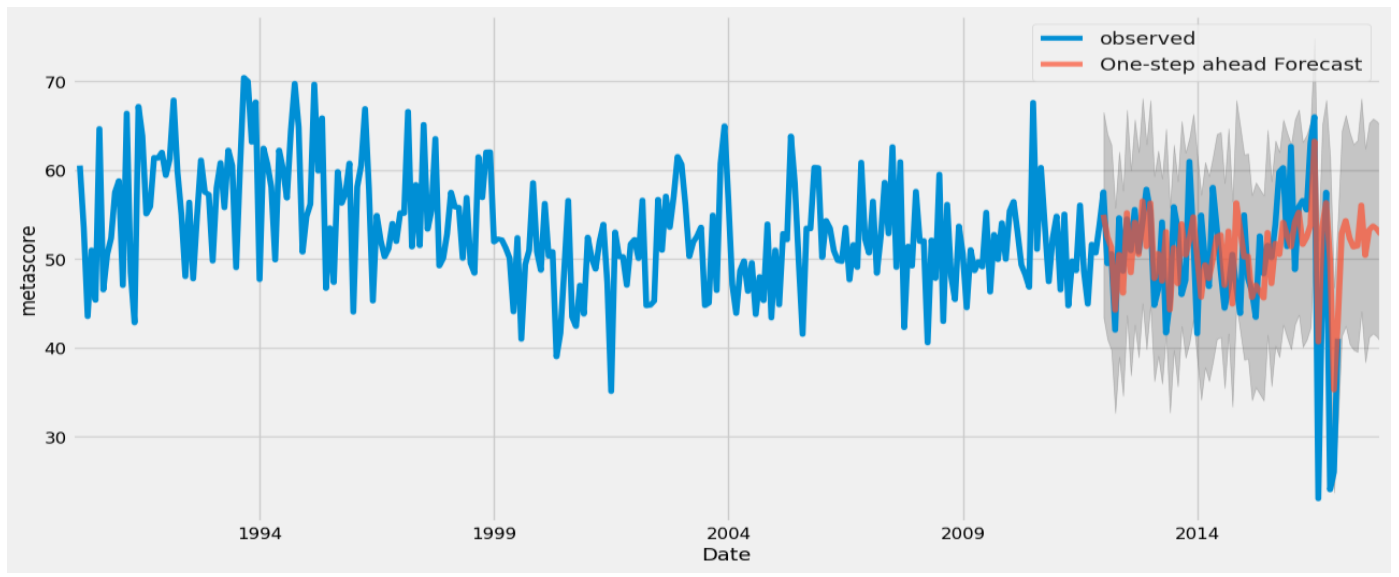

Figure 10. Forecasted value compared to actual value residual plot

These statistical tests to determine its patterns and characteristics as mentioned above include the Augmented Dickey-Fuller Test for stationarity and analysis of the ACF and PACF plots for the autocorrelation and moving average parameters. From the results obtained from these tests along with a comparison of the AIC values the time series model to be used is finalized as the Seasonal Auto-Regressive Integrated Moving Average with eXogenous factors model, or in short, the 
SARIMAX model. We can see that the model gives us a roughly accurate result for the forecast. We plot the same for our 2020 dataset, using the SARIMAX model we used before to get similar outputs as in Figure 11.

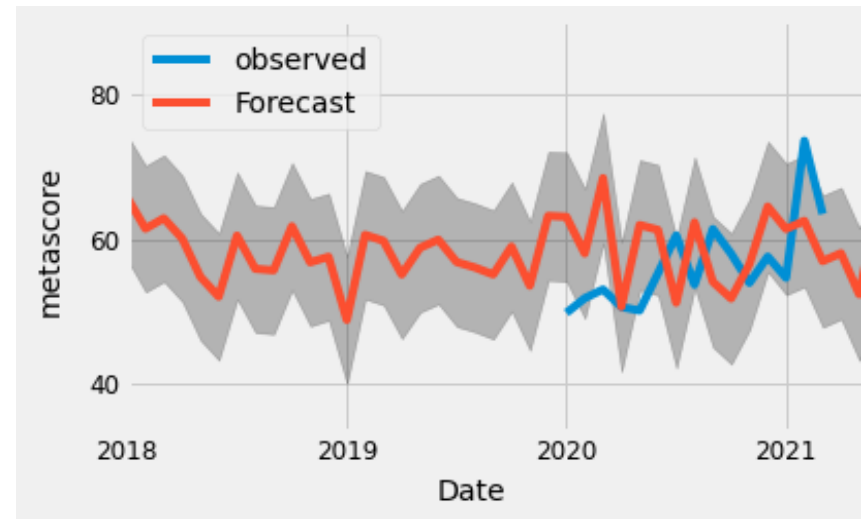

Figure 11. Observed vs forecasted values

\section{Artificial Neural Network}

The Artificial Neural Network is the most complex model used for prediction, and here, we make use of the Multi-Layer Perceptron network for our classification. The training data is fed into the model and run over multiple epochs. .The model gives us an accuracy of $86.16 \%$ and on plotting the loss curve, we notice a gradual decline in the loss percentage, which indicates a rapid improvement in performance after every epoch. Steps to reduce overfitting have also been taken. These include limiting the maximum number of iterations and implementing Early Stopping. The loss curve for the model is displayed in Figure 12. On testing the model with our validation 2020 dataset, we obtain a final accuracy of $88.056 \%$.

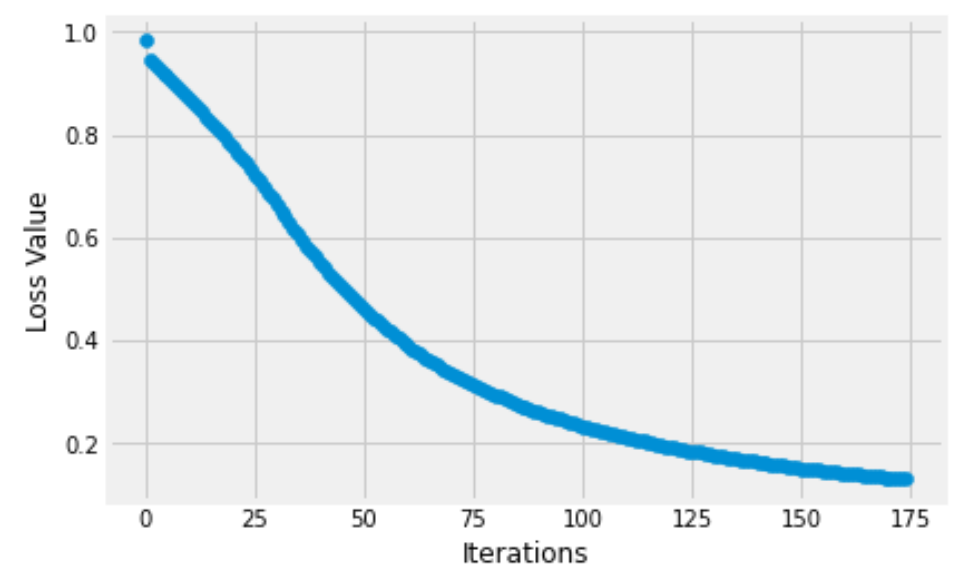

Figure 12. Loss Curve for the ANN 


\section{RESULTS AND INTERPRETATION OF VALUES}

\subsection{Regression}

Wald's test for Logistic Regression proved the statistical significance of the model in Table 1. Wald's test is used to check if each of the variables involved in Logistic Regression will give statistically significant results, indicated by the p-values depicted below. The Chi-square tests, though high in number for a couple of attributes, on the whole, proves that the Logistic regression model is still significantly valid in terms of the results we obtain from it.

Table 1. Wald's Test for Logistic Regression

\begin{tabular}{|c|c|c|}
\hline $\mathbf{X}$ & $\mathbf{C h i}^{\wedge} \mathbf{2}$ & $\mathbf{p - v a l u e ~} \mathbf{\text { Chi`2 }}$ \\
\hline const & 1062.614071 & $4.411953 \mathrm{e}-233$ \\
\hline top1000_voters_rating & 1104.475166 & $3.517505 \mathrm{e}-242$ \\
\hline Action & 53.033523 & $3.279040 \mathrm{e}-13$ \\
\hline Crime & 22.853796 & $1.748038 \mathrm{e}-06$ \\
\hline Drama & 24.430425 & $7.704234 \mathrm{e}-07$ \\
\hline Fantasy & 16.520951 & $4.811547 \mathrm{e}-05$ \\
\hline Mystery & 25.968786 & $3.469824 \mathrm{e}-07$ \\
\hline Romance & 3.914710 & $4.786528 \mathrm{e}-02$ \\
\hline Sport & 7.101317 & $7.702733 \mathrm{e}-03$ \\
\hline Thriller & 9.629585 & $1.914678 \mathrm{e}-03$ \\
\hline War & 4.919469 & $2.655568 \mathrm{e}-02$ \\
\hline
\end{tabular}

On studying these values, we shift our investigation to the other regression models as well, displayed in Table 2. The Durbin Watson Test is a hypothesis test that checks for autocorrelation between error terms. In this test, the null hypothesis indicates that there is no autocorrelation present in the data used for prediction. A Durbin-Watson statistic that is close to 2 implies no autocorrelation. This is the case for all our regression models since it has Durbin-Watson statistic values ranging from 1.7163 as in the case of LASSO regression to 1.963 for Multiple Linear Regression. Multiple Linear Regression shows the highest value in terms of the R-square statistic, which is proved by the Analysis of Variance test (or the F-statistic). This is also a hypothesis test where the null hypothesis indicates that all regression coefficients for the model should ideally be zero. Hence it is a test that ensures the overall regression of the model. In Multiple Linear Regression, the F-statistic is high unlike that for Ridge and Lasso Regression indicating that the results are not significant. However, the F-statistic value is significantly higher for SLR which seems to give it along with MLR more credit compared to the other Regressions in the ranking of models as its R2 value was only marginally lower. The Jarque-Bera test is a goodness-of-fit test of whether sample data has the skewness and kurtosis matching a normal distribution. Lagrange Multiplier test (LM test also known as Breusch Godfrey test) is a hypothesis test for autocorrelation in the errors in a regression model, the null hypothesis being the absence of autocorrelation. Hence, in terms of the normal distribution of errors, all regressions except SLR are statistically significant with respect to their Jarque-Bera and Lagrange Multiplier values. Therefore, it can be interpreted that out of these regressions only MLR and Logistic Regression can go for further analysis since they are statistically significant for all parameters. 
Table 2. Statistical tests for Regression Analysis

\begin{tabular}{|c|c|c|c|c|}
\hline $\mathbf{X}$ & $\begin{array}{c}\text { Simple } \\
\text { Linear }\end{array}$ & $\begin{array}{c}\text { Multiple } \\
\text { Linear }\end{array}$ & Ridge & LASSO \\
\hline R2/ Pseudo R2 & 0.556 & 0.619 & 0.4855 & 0.46 \\
\hline Adjusted R-Square & 0.556 & 0.618 & 0.48 & 0.4553 \\
\hline F-Statistics & 6007 & 485.0 & $2.449 \mathrm{e}-37$ & $2.477 \mathrm{e}-27$ \\
\hline Durbin-Watson Test & 1.952 & 1.963 & 1.79127 & 1.7163 \\
\hline Jarque-Bera (JB) Test & 9.617 & 0.91 & 34.778 & 90.18286 \\
\hline $\begin{array}{c}\text { Lagrange Multiplier } \\
\text { Statistic }\end{array}$ & 14.237 & 145.0 & 208.301 & 185.15 \\
\hline Accuracy & 0.71 & 0.711 & 0.72 & 0.72 \\
\hline
\end{tabular}

\subsection{Classification}

The parameters have been tuned to obtain the maximum Silhouette Score which ranges from - 1 to 1 and a higher Silhouette Score for a given model indicates better cluster formation within that model. In terms of the Silhouette Score, it is observed that K-Means has a very good performance indicating that the clusters formed are well-defined and separated. However, its accuracy is low which shows that although there is good separability it doesn't correspond with the correct metascore values very well. On the other hand, SVM shows a comparatively better accuracy with less efficient cluster formation as compared to K-Means. The relatively poor performance of K-Means shows that unsupervised learning may not be a good approach to solve the movie success prediction problem.

Table 3. Statistical tests for Classification Analysis

\begin{tabular}{|c|c|c|}
\hline $\mathbf{X}$ & K-Means & SVM \\
\hline Silhouette Test & 0.7021 & 0.13387 \\
\hline Accuracy & 0.49934 & 0.71 \\
\hline
\end{tabular}

\subsection{Time Series Analysis}

From the autocorrelation and partial autocorrelation plot results, it is evident that the AR and MA parameters of the Time Series to be considered should be 1 each. This is supported by the Durbin Watson Statistic being 1.4928194722663908 which indicates positive autocorrelation. The Augmented Dickey Fuller Test was showing stationarity based on the value given. The presence of seasonality was confirmed when the model with seasonality was giving a lower AIC value. The presence of exogenous variables was confirmed when they resulted in a reduction in the RMS value. The model was tuned to get the lowest possible Likelihood, AIC, BIC and HQIC. The Ljung Box Statistic leading to a p-value greater than the significance level also shows the validity of the model. The Jarque-Bera Statistic confirms the heteroscedasticity.

Table 4. Time Series Analysis Statistical Test Observations

\begin{tabular}{|c|c|}
\hline $\mathbf{X}$ & Older movies analysis \\
\hline Autocorrelation & Cuts of to 0 after 1 lag \\
\hline Partial Autocorrelation & Cuts of to 0 after 1 lag \\
\hline Augmented Dickey-Fuller Test Statistic & -10.462528698062133 \\
\hline RMSE & 62.19 \\
\hline Log-Likelihood & -965.539 \\
\hline AIC & 1949.079 \\
\hline BIC & 1982.679 \\
\hline
\end{tabular}


International Journal of Artificial Intelligence and Applications (IJAIA), Vol.13, No.1, January 2022

\begin{tabular}{|c|c|}
\hline HQIC & 1962.512 \\
\hline Ljung-Box (Q) & 24.64 \\
\hline Skewness & -0.26 \\
\hline Kurtosis & 4.13 \\
\hline Jarque-Bera (JB) Test & 19.9 \\
\hline Heteroscedasticity & 0.95 \\
\hline Durbin-Watson Test & 1.4928194722663908 \\
\hline
\end{tabular}

\subsection{Artificial Neural Network}

The neural network shows its superiority by giving a very high accuracy with minimum possible loss. This is done by tuning the hyper-parameters which has helped improve the loss curve over multiple iterations for the prediction. It also allows for the reduction of overfitting. The list of attributes and parameters are given below.

Table 5. Artificial Neural Network

\begin{tabular}{|c|c|}
\hline Attributes & $\begin{array}{c}\text { 'duration', 'avg_vote', 'Action', 'Adventure', 'Animation', 'Biography', } \\
\text { 'Comedy', 'Crime', 'Drama', 'Family', 'Fantasy', 'Horror', 'Mystery', 'Thriller' }\end{array}$ \\
\hline Type & Multi-layer Perceptron Classifier \\
\hline Architecture & $\begin{array}{c}\text { İnput Layer: 14 } \\
\text { Hidden Layer: } 100 \\
\text { Output Layer: } 3\end{array}$ \\
\hline Output Type & Ternary \\
\hline Initial Loss & 0.6915657421532461 \\
\hline Final Loss & 0.1624720046108523 \\
\hline Activation Function & Logistic \\
\hline Optimizer & Adam \\
\hline Early Stopping & True \\
\hline Validation Fraction & 0.1 \\
\hline $\begin{array}{c}\text { Number of training } \\
\text { examples }\end{array}$ & 4796 \\
\hline Loss Curve Type & Strictly Decreasing \\
\hline Testing results with & $93.055555556 \%$ \\
2020 dataset & \\
\hline
\end{tabular}

\subsection{Comparison of Valid Models with Similar Accuracy}

SLR and Ridge and Lasso Regression were not considered as they proved to be statistically insignificant by the normality test and F Statistic respectively. The 3 models shown in Table 5 have comparable accuracy. A Jaccard Index which is used to find the similarity between two sets of attributes, in this case, is obtained by comparing the attributes of the model compared to what was available in the testing dataset. This is used as an indication of the extent to which attributes used in the model are useful for data available for movies released at present. From this, it can be concluded that SVM is the better modelamong the 3 followed by Multiple Linear Regression and Logistic Regression respectively. 
International Journal of Artificial Intelligence and Applications (IJAIA), Vol.13, No.1, January 2022

Table 6. Comparison of Models with Similar Accuracy

\begin{tabular}{|c|c|c|c|}
\hline $\begin{array}{l}\text { Model } \\
\text { Name }\end{array}$ & Attributes & Attributes 2020 & $\begin{array}{c}\text { Jaccard } \\
\text { Index }\end{array}$ \\
\hline $\begin{array}{l}\text { Multiple } \\
\text { Linear } \\
\text { regression }\end{array}$ & $\begin{array}{c}\text { 'budget','reviews_from_users','review_fr } \\
\text { om_critics', } \\
\text { 'top1000_voters_ratings', } \\
\text { 'Action','Animation','Crime', } \\
\text { 'Drama','Family','Fantasy', 'Horror', } \\
\text { 'Music', 'Musical', } \\
\text { 'Mystery','Sport','Thriller' }\end{array}$ & $\begin{array}{l}\text { 'duration','Action','Animati } \\
\text { on','Biography','Drama', } \\
\text { 'Horror' }\end{array}$ & $\begin{array}{c}4 / 18=0.22 \\
2\end{array}$ \\
\hline $\begin{array}{l}\text { Logistic } \\
\text { regression }\end{array}$ & $\begin{array}{l}\text { 'top1000_voters_rating', } \\
\text { 'Action','Crime','Drama', } \\
\text { 'Fantasy','Mystery','Romance', 'Sport', } \\
\text { 'Thriller,' 'War' }\end{array}$ & $\begin{array}{c}\text { 'avg_vote','Action','Crime', } \\
\text { 'Fantasy','Mystery' }\end{array}$ & $4 / 11=0.18$ \\
\hline SVM & $\begin{array}{c}\text { 'top1000_voters_rating', 'Action', 'Crime', } \\
\text { 'Drama', 'Fantasy', 'Mystery','Romance', } \\
\text { 'Sport','Thriller', 'War' }\end{array}$ & $\begin{array}{c}\text { 'avg_vote', 'Action','Crime', } \\
\text { 'Drama','Fantasy','Mystery',' } \\
\text { Thriller' }\end{array}$ & $\begin{array}{c}6 / 11=0.54 \\
5\end{array}$ \\
\hline
\end{tabular}

\subsection{Some Movie Success Prediction Examples}

Table 7 shows all the model results for the most recent movies in the testing dataset used. This is given to show the variation in predictions made by each individual model with respect to the data given to them for the classification purpose. Though we cannot make conclusions directly from these results, we can make simple assumptions based on how close the classification of movies is for each model. As shown below, all the models do not give the same output. Here $\mathrm{H}$ stands for Hit, F stands for Flop and N stands for Neutral i.e. mediocre.

Table 7. Movie Success Prediction Examples

\begin{tabular}{|l|c|c|c|c|c|c|c|c|c|}
\hline Movie Name & $\begin{array}{l}\text { True } \\
\text { Success }\end{array}$ & SLR & MLR & KMeans & Logistic & Ridge & Lasso & SVM & ANN \\
\hline Jeanne & F & N & N & N & N & N & N & N & N \\
\hline $\begin{array}{l}\text { I Trapped the } \\
\text { Devil }\end{array}$ & N & N & N & N & F & N & N & N & H \\
\hline Midsommar & H & N & N & N & F & N & N & N & H \\
\hline Knives Out & H & H & H & N & N & H & H & H & N \\
\hline Sextuplets & F & N & N & N & F & N & N & N & H \\
\hline Unplanned & F & N & F & N & F & F & F & F & F \\
\hline $\begin{array}{l}\text { Cold Blood } \\
\text { Legacy }\end{array}$ & F & N & F & N & F & N & F & N & F \\
\hline $\begin{array}{l}\text { Playing with } \\
\text { Fire }\end{array}$ & F & H & F & N & F & F & F & F & H \\
\hline Jexi & N & N & N & N & F & N & F & N & H \\
\hline Tomasso & N & N & N & N & F & N & N & N & N \\
\hline
\end{tabular}




\subsection{Role of Attributes in the Prediction and Classification}

Not all features that were a part of the data that was collected were used in each of the models. So, it is important to know what kind of features influence the success of a movie. The features like the duration of a movie, average vote, budget, reviews from users and reviews from critics were used in only one of the models - duration and average vote being in the artificial neural network and budget and the review related features being for multiple linear regression. However, the attribute "top_1000_voters_rating" seems to appear in many models. This shows that among all the attributes that could have been influencing the success of a movie these attributes seem to be playing a comparatively major role, the "top_1000_voters_rating" being more important. Also, it is seen that from the 21 genres that a movie can belong to, only some of them end up contributing to the models with statistical significance. So it can be said that only a particular set of genres seem to contribute to the fate of a movie. And this may indicate that some genres are more popular among the audiences compared to others. These include genres such as "Action", "Crime", "Drama", "Fantasy", "Mystery" and "Thriller" that appear in all the models and therefore may be considered as the set of genres that contribute to the future of a movie.

\section{DISCUSSION}

Our literature survey opened up a lot of avenues into various concepts of Machine Learning techniques used to predict how successful a movie truly is. The inspiration for our paper comes from the fact that despite these models giving substantial performances, there was no proof of the reliability of the results obtained. Though we have explored techniques with low efficiency, the results it bears hold a certain level of significance that can be used for further research. This was achieved through performing tests such as normal distribution of errors in regression models, coherence of clusters, the accuracy of forecasting values, and many more based on each model we studied. This ensured the removal of any form of inconsistency from the models like bias, variance, overfitting or underfitting, and so on. To provide corroboration to our statistical proofs, we scraped freshly obtained movies from the IMDb website from the year 2020. This paper has shown that data and its attributes play a more vital role compared to the models that we use. Choosing the right set of attributes provides useful insights and authentic results. Though we have not considered attributes such as language, actors, directors and so on, we can see how some features such as the genre of a movie and the number of votes play an important role in the prediction process, which gives room for further investigation for a larger variety of attributes to be studied this way.

\section{CONCLUSION}

It is seen that movie success can be predicted using various approaches and each of these make use of different kinds of features. Among these models, the Artificial Neural Network performs the best and only makes use of the duration of the movie and its average vote apart from the genre. Following that there are models such as Multiple Linear Regression, Logistic Regression and Support Vector Machine that have similar accuracies which is the highest apart from the Artificial Neural Network. Here, Support Vector Machine is considered to be a slightly better model as compared to Multiple Linear Regression and Logistic Regression because it has the most attribute availability concerning proof was, the data obtained for the testing dataset. The final valid model that comes in the ranks is K-Means having a very low accuracy despite showing a good clustering. This indicates that a supervised learning approach works better for the given problem statement. The Regularization models and Simple Linear Regression are deemed invalid because they are not statistically significant. The SARIMAX model that is used for time-series forecasting provides very good results with low error values. When looking at the attributes that 
were used for prediction it is seen that the "top1000_voters_rating" is important and influential as it is used in almost all models and some genres predict better than others.

\section{FUTURE WORK}

In the near future, we intend to investigate the various enhancements that can be made to individual models in order to improve their performance, and thus broaden our perspective on the classification of a variety of other models with comparable performances in order to demonstrate the significance of each one. This analysis has not used attributes that are categorical and has a lot of examples that can be categories such as the actors, directors of the movies and so on. As a result, researching the influence of these qualities might be another area for future research. Another feature that may be investigated is the movie descriptions, which can be done using advanced Natural Language Processing algorithms.

\section{REFERENCES}

[1] Abidi, S.M.R., Xu, Y., Ni, J. et al. Popularity prediction of movies: from statistical modeling to machine learning techniques.Multimed Tools Appl79, 35583-35617 (2020). (Abidi et al. 2020)

[2] IMDb extensive dataset, Kaggle: https://www.kaggle.com/stefanoleone992/imdb-extensive-dataset

[3] Ahmad J., Duraisamy P., A. Yousef and Buckles, B.: Movie success prediction using data mining, pp. 1-4. In: 2017 8th International Conference on Computing, Communication and Networking Technologies (ICCCNT), doi: 10.1109/ICCCNT.2017.8204173. (Ahmad et al. 2017) Delhi, India (2017)

[4] Nithin, Vr, M. Pranav, PbSarath Babu and A. Lijiya. "Predicting Movie Success Based on IMDB Data." International journal of business 003 (2014): 34-36.(Bristi et al. 2019; Subramaniyaswamy et al. 2017; Nithin et al. 2014) (2014)

[5] Subramaniyaswamy V., Vaibhav M. V., Prasad R. V. and Logesh R.: Predicting movie box office success using multiple regression and SVM, pp. 182-186. In: 2017 International Conference on Intelligent Sustainable Systems (ICISS), doi: 10.1109/ISS1.2017.8389394. (Bristi et al. 2019; Subramaniyaswamy et al. 2017)Palladam (2017)

[6] Lee K, Park J, Kim I \& Choi Y: Predicting movie success with machine learning techniques: ways to improve accuracy. In: Information Systems Frontiers, vol (in press), doi: 10.1007/s10796-016-9689-z (Lee et al. 2018)(2016)

[7] Darapaneni N. et al.: Movie Success Prediction Using ML, pp. 0869-0874. In: 2020 11th IEEE Annual Ubiquitous Computing, Electronics \& Mobile Communication Conference (UEMCON), doi: 10.1109/ UEMCON51285.2020.9298145. (Darapaneni et al. 2020) New York City, NY (2020)

[8] Quader N., Gani M. O., Chaki D. and Ali M. H.: A machine learning approach to predict movie boxoffice success. In: 2017 20th International Conference of Computer and Information Technology (ICCIT), pp. 1-7, doi: 10.1109/ICCITECHN.2017.8281839. (Quader et al. 2017) Dhaka (2017)

[9] Dhir R. and Raj A.: Movie Success Prediction using Machine Learning Algorithms and their Comparison, pp. 385-390. In: 2018 First International Conference on Secure Cyber Computing and Communication (ICSCCC), doi: 10.1109/ICSCCC.2018.8703320 (Dhir and Raj 2018) Jalandhar, India (2018)

[10] Ericson, J., \&Grodman, J.: A predictor for movie success. CS229, Stanford University.(2013)

[11] Quader N., Gani M. O. and Chaki D.: Performance evaluation of seven machine learning classification techniques for movie box office success prediction. In: 2017 3rd International Conference on Electrical Information and Communication Technology (EICT), pp. 1-6, doi: 10.1109/EICT.2017.8275242. (Quader et al. 2017; Quader et al. 2017) Khulna (2017)

[12] Kumar, U.D., Wiley, Business Analytics: The Science of Data-Driven Decision Making(2017).

[13] Navidi, W., McGraw Hill: Statistics for Engineers and Scientists:Indian Edition(2011)

[14] Sharma, T., Dichwalkar, R., Milkhe, S., and Gawande, K.: Movie Buzz - Movie Success Prediction System Using Machine Learning Model. In: 2020 3rd International Conference on Intelligent Sustainable Systems (ICISS), pp. 111-118, doi: 10.1109/ICISS49785.2020.9316087. Thoothukudi, India (2020) (Sharma et al. 2020)

[15] Multi-Label Binarizer Python Documentation: MultiLabelBinarizer 
International Journal of Artificial Intelligence and Applications (IJAIA), Vol.13, No.1, January 2022

[16] Verma, G., and Verma, H.: Predicting Bollywood Movies Success Using Machine Learning Technique, pp. 102-105. In: 2019 Amity International Conference on Artificial Intelligence (AICAI), doi: 10.1109/AICAI.2019.8701239. (Verma and Verma 2019) Dubai, United Arab Emirates, (2019)

[17] Lash, Michael T., and Kang Zhao. "Early predictions of movie success: The who, what, and when of profitability." Journal of Management Information Systems 33, no. 3 (2016): 874-903. (Lash and Zhao 2016)

[18] Na, S., Xumin, L., and Yong, G.: Research on k-means Clustering Algorithm: An Improved k-means Clustering Algorithm, pp. 63-67. In: 2010 Third International Symposium on Intelligent Information Technology and Security Informatics, doi: 10.1109/IITSI.2010.74 (Na et al. 2010)Jinggangshan, (2010)

[19] Bristi, W. R., Zaman, Z., and Sultana, N.: Predicting IMDb Rating of Movies by Machine Learning Techniques, pp. 1-5. In: 2019 10th International Conference on Computing, Communication and Networking Technologies (ICCCNT), doi: 10.1109/ICCCNT45670.2019.8944604. (Bristi et al. 2019)Kanpur, India, (2019)

[20] S. F. Ershad and S. Hashemi, "To increase quality of feature reduction approaches based on processing input datasets," 2011 IEEE 3rd International Conference on Communication Software and Networks, 2011, pp. 367-371, doi: 10.1109/ICCSN.2011.6014289. (Ershad and Hashemi 2011)

[21] Fekri-Ershad, S. (2019). Gender classification in human face images for smartphone applications based on local texture information and evaluated Kullback-Leibler divergence. Traitement du Signal, Vol. 36, No. 6, pp. 507-514. (Fekri-Ershad 2019)

[22] R. Dhir and A. Raj, "Movie Success Prediction using Machine Learning Algorithms and their Comparison," 2018 First International Conference on Secure Cyber Computing and Communication (ICSCCC), 2018, pp. 385-390, doi: 10.1109/ICSCCC.2018.8703320. (Dhir and Raj 2018)

[23] Ahmad, Ibrahim Said, et al. "A survey on machine learning techniques in movie revenue prediction." SN Computer Science 1.4 (2020): 1-14. (Ahmad et al. 2020)

[24] Agarwal M, Venugopal S, Kashyap R, Bharathi R (2021) "A comprehensive study on various statistical techniques for prediction of movie success", 2nd International Conference of Machine Learning Techniques and Data Science, doi: 10.5121/csit.2021.111802 (Agarwal et al. 2021)

[25] N. Darapaneni et al., "Movie Success Prediction Using ML," 2020 11th IEEE Annual Ubiquitous Computing, Electronics \& Mobile Communication Conference (UEMCON), 2020, pp. 0869-0874, doi: 10.1109/UEMCON51285.2020.9298145. (Darapaneni et al. 2020)

[26] Ankit, M. Lakshmi, K. A. Shastry, A. Sandilya and R. Shekhar, "A comparative analysis of Machine Learning approaches for Movie Success Prediction," 2020 Fourth International Conference on ISMAC (IoT in Social, Mobile, Analytics and Cloud) (I-SMAC), 2020, pp. 684-689, doi: 10.1109/ISMAC49090.2020.9243589. (Ankit et al. 2020)

[27] W. M. D. R. Ruwantha, K. Banujan and K. Btgs, "LSTM and Ensemble Based Approach for Predicting the Success of Movies Using Metadata and Social Media," 2021 International Conference on Innovation and Intelligence for Informatics, Computing, and Technologies (3ICT), 2021, pp. 626630, doi: 10.1109/3ICT53449.2021.9581601. (Ruwantha et al. 2021) 
International Journal of Artificial Intelligence and Applications (IJAIA), Vol.13, No.1, January 2022

\section{AUTHORS}

Shreya Venugopal, Student at PES university pursuing B-Tech in Computer Science, member of the ACM student chapter, specialization in Machine Intelligence and Data Science and an avid coder.

Rishab Kashyap, Student at PES University pursuing B-Tech in Computer Science, member of the ACM student chapter, Specialization in Machine Intelligence and Data Science along with minors degree in Electronics and Communication.

Manav Agarwal, Undergraduate Student at PES university pursuing B-Tech in Computer Science, Specialization in Machine Intelligence and Data Science with an innate interest in gadgets and electronics.

R Bharathi, Associate professor at PES University specialisation in Data Science, Machine learning and Data Analytics. 